\title{
A FAST MODEL-PREDICTIVE SPEED CONTROLLER FOR MINIMISED CHARGE CONSUMPTION OF ELECTRIC VEHICLES
}

\author{
Tim Schwickart, Holger Voos, Jean-Régis Hadji-Minaglou, and Mohamed Darouach
}

\begin{abstract}
This paper presents the design of a real-time implementable energy-efficient model-predictive cruise controller for electric vehicles including the driving speed reference generation. The controller is designed to meet the properties of a series-production electric vehicle whose characteristics are identified and validated by measurements. The predictive eco-cruise controller aims at finding the best compromise between speed-reference tracking and energy consumption of the vehicle using an underlying dynamic model of the vehicle motion and charge consumption. The originally non-linear motion model is transformed into a linear model mainly by using a coordinate transform. To obtain a piecewise linear approximation of the charge consumption map, the measured characteristics are approximated by a convex piecewise linear function represented as the maximum of a set of linear constraint functions. The reformulations finally lead to a model-predictive control approach with quadratic cost function, linear prediction model and linear constraints that corresponds to a piecewise linear system behaviour and allows a fast real-time implementation with guaranteed convergence. Simulation results of the closed-loop operation finally illustrate the effectiveness of the approach.
\end{abstract}

Key Words: Driver assistance systems, model-predictive control, optimal control, electric vehicles, energy efficiency.

\section{INTRODUCTION}

The cruising range is one of the most decisive drawbacks of electric vehicles and an important problem that needs to be solved in electric mobility. Since the on board (tank-to-wheel) efficiency of electric vehicles can hardly be improved, there are only two possibilities to increase the range. The first is the improvement of the battery technology towards higher capacities and lower weights. The second measure is to address the driving style that has a huge influence on the energy consumption of a vehicle [1]. Due to possible savings of 10 to $20 \%$, it is a promising approach to adapt and optimise the driving style predictively according to the road topology and the traffic situation in order to save energy [2].

A sophisticated way to address this problem is controlling the driving speed automatically by a driver-assistance system (eco-cruise control). Eco-cruise

Manuscript received July 31, 2014; revised January 26, 2015; accepted September 10,2015 .

Tim Schwickart (corresponding author, e-mail: tim.schwickart@uni.lu) and Holger Voos are with the Interdisciplinary Centre for Security, Reliability and Trust (SnT), University of Luxembourg, 6 rue Coudenhove-Kalergi, L-1359 Luxembourg, Luxembourg.

Jean-Régis Hadji-Minaglou is with the Faculty of Science, Technology and Communication (FSTC), University of Luxembourg, 6 rue Coudenhove-Kalergi, L-1359 Luxembourg, Luxembourg.

Mohamed Darouach is with the Centre de Recherche en Automatique de Nancy (CRAN) UMR-CNRS 7039, Université de Lorraine, IUT de Longwy, 186 rue de Lorraine, F-54400 Cosnes et Romain, France. control can be described as an optimal control problem $[3,4]$. The accelerator and brake pedal positions are the control inputs of the system while the driving speed and the energy consumption are given by an underlying dynamic vehicle model (based on the previous knowledge of the speed limits and the road slope). The controller outputs are the optimisation variables that minimise a cost function containing terms related to driving speed and energy consumption. As the car is running under changing traffic and environment conditions, it is hardly possible to calculate and apply the complete optimal driving strategy in advance. A suitable approach to overcome this problem is to apply model-predictive control (MPC) in a receding horizon fashion, where the optimisation is carried out for a finite prediction horizon and is repeated at every time step. This control strategy has been considered as the tool of choice for the eco-cruise control of fuel-powered cars in several works [5-8]. Recently, eco-cruise control for purely electric vehicles has been considered in [9-12]. The biggest challenge in the application of MPC is the requirement of a fast online-optimisation which is hampering a real-time implementation. Therefore, the formulation of the optimal control problem is decisively important in order to achieve a fast solution. The most desirable formulation comprises a quadratic cost function and linear constraints including a linear dynamic plant model, since efficient solvers with guaranteed convergence are available for the resulting discretised quadratic optimisation problem. 
However, an overall linearisation of the vehicle dynamics around one operation point is not satisfactory since the prediction has to be carried out over a wide range of operating points whereas a linearisation only yields good results in the area close to the operating point. Previous works use analytical solutions of the non-linear optimal control problem based on Pontryagin's Maximum Principle (indirect methods) $[3,4]$ or alternatively efficient discretisation techniques to solve the non-linear optimisation problem directly $[5,7,13]$. Using analytical solutions however, the optimal controller cannot be designed in a flexible way since no constraints on the state variables, dynamically changing weightings or measured disturbances can be considered. On the other hand, the numerical methods for non-linear optimisation do not guarantee a (fast) convergence of the optimisation algorithm.

The contribution of this paper is a predictive energy-efficient control formulation especially for electric vehicles in the form a quadratic optimal control problem with linear constraints. The linear dynamic model is obtained by reformulations of the equations and an exploitation of the optimisation problem setup instead of an overall linearisation. Thus, the non-linearities are considered implicitly by the control system while the results are equivalent to the aforementioned non-linear approaches. In contrast to these, the quadratic optimisation can be solved in real-time with much lower computational effort.

The paper extends the basic idea of the model-predictive eco-cruise controller presented in $[14,15]$ designed especially for an electric car by a more sophisticated model and a speed reference generator.

\section{OVERALL SYSTEM SETUP}

The overall system is planned to work as a driver-assistance system mainly in highway and overland driving. It shall control the speed automatically with respect to

- the road curvature,

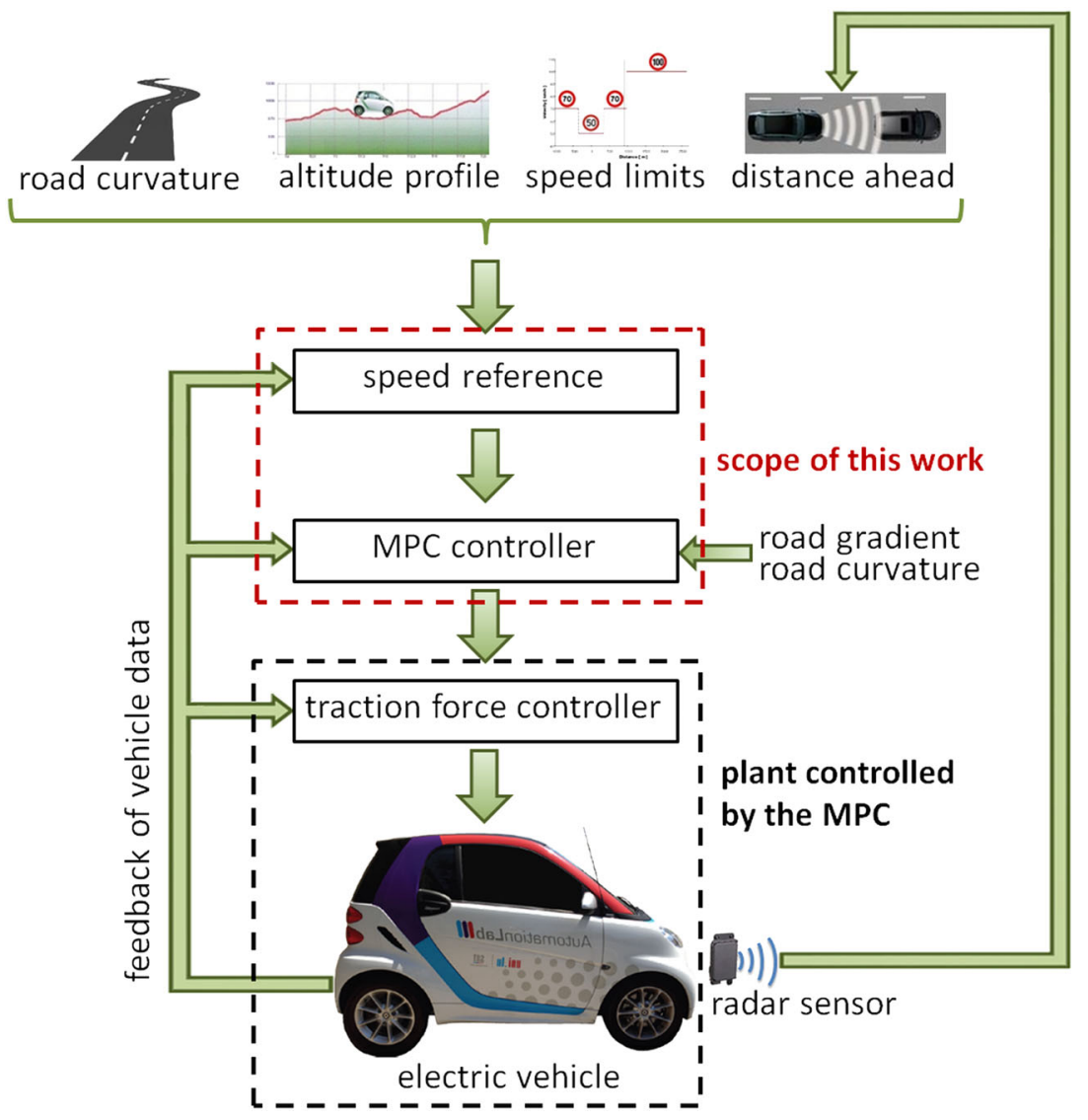

Fig. 1. Predictive cruise control system for energy-efficient driving. 
- the road slope angle,

- the speed limits and

- the distance to the preceding vehicle

similar to adaptive cruise control (ACC) solutions that are already available in modern cars. In contrast to this, the proposed control system is based on an online-optimisation with a strong focus on energy efficiency of electric vehicles. In order to adapt our model and the control approach to a realistic use case, we consider a series-production Smart Electric Drive (ED) which is available in our laboratory (see Fig. 1).

To achieve the desired predictive behaviour, first a speed-reference trajectory is generated from digital maps of the road ahead and sensor information. The reference generator provides the speed set-point trajectory with respect to speed limits, the distance to the preceding car and the road curvature. While curvature, altitude profile and speed limits can be read from a car navigation system, the distance to the preceding car is measured by a radar sensor as commonly used in existing adaptive cruise control realisations. The actual driving state of the car is represented by the driving speed and the state of charge of the battery. Given this information, the model-predictive cruise controller aims at finding a traction force trajectory leading to an optimal trade-off between speed reference tracking and minimisation of the charge consumption. Since the traction force cannot directly serve as control input to the vehicle, a subsidiary controller regulates the traction force by actuating the accelerator pedal. The boosting ability of the Smart ED at fully pressing the accelerator (including the kickdown switch below the pedal) as well as the brake pedal are not planned to be actuated in this setup. The structure of the overall control system is given in Fig. 1.

This paper focuses on the design of the MPC controller and the reference generator. The subsidiary traction force controller is assumed to work ideally, here. The battery management is done by the subsidiary internal control system of the vehicle which avoids unsafe operation. The proposed MPC controller influences the battery current by controlling the accelerator pedal position like a human driver would do.

\section{DYNAMIC MODEL OF THE ELECTRIC VEHICLE}

A suitable model needs to be adapted to the dynamic behaviour of our experimental car, a Smart $E D$, whose centre-piece is a permanent magnet synchronous machine. This is a three-phase AC machine that is able to work as motor or generator allowing energy recovery when decelerating. A lithium-ion battery serves as accumulator and supplies the synchronous machine via a DC/AC converter. The rear wheels are driven by the motor through a gear box with one fixed transmission ratio.

The model is subdivided according to its outputs of interest, namely a model of the driving speed $v$ (Section 3.1) and a model of the charge consumption $C_{e l}$ of the vehicle (Section 3.2). The model input (and control input) is the traction force at the wheels $F_{t r a c}$.

\subsection{Model of the longitudinal dynamics}

The common approach to model the longitudinal dynamics of a vehicle is to describe a one-dimensional motion of a point mass based on Newton's second law $\sum F=m \cdot \frac{d v}{d t}$. The main forces acting in longitudinal direction on the vehicle are the traction force $F_{\text {trac }}$ as well as the driving resistance forces [16]:

- air drag resistance $F_{d}$,

- rolling resistance $F_{r}$,

- curvature resistance $F_{r c}$

- and grade resistance $F_{g r}$.

A diagram of the forces acting in longitudinal direction is given in Fig. 2. The equations of the driving resistance forces are listed in the following.

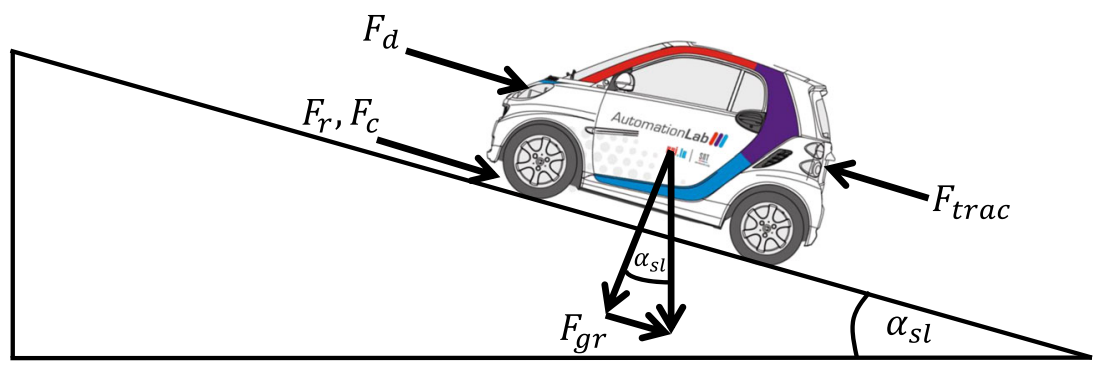

Fig. 2. Forces acting on the vehicle in longitudinal direction. 


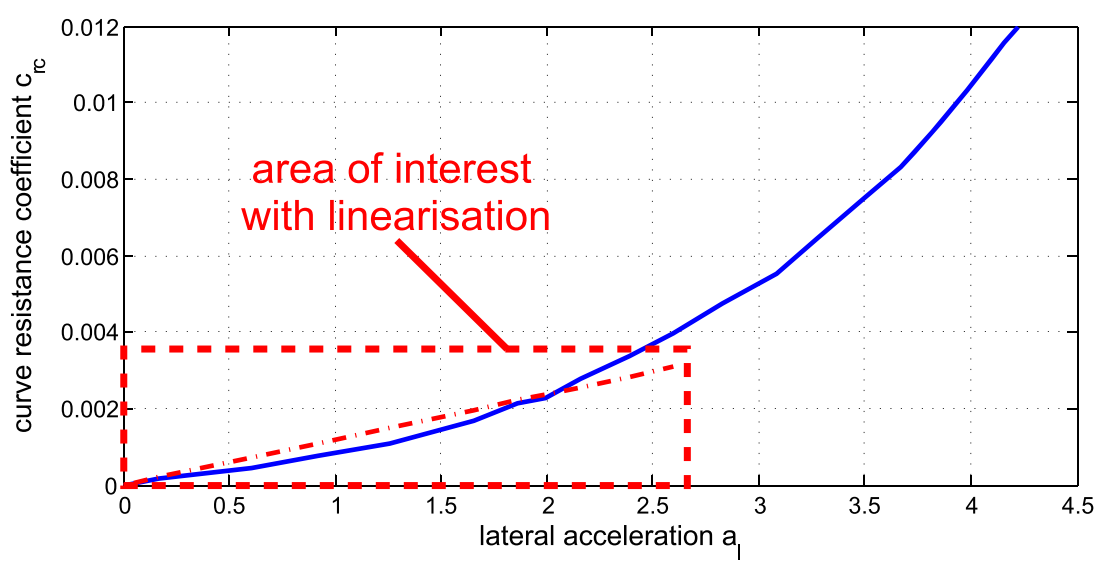

Fig. 3. Relation between the curve resistance coefficient $c_{r c}$ and the lateral acceleration acting on the vehicle $a_{l}$ for a tyre of dimensions 195/65V15, edited from [17].

The rolling resistance force $F_{r}$ is a function of the road slope angle $\alpha_{s l}$. The parameters are the vehicle kerb weight $m_{v}$, the payload $m_{l}$, the gravitational constant $g$ and the rolling resistance coefficient of the tyres $c_{r}[16]$.

$$
F_{r}=\left(m_{v}+m_{l}\right) \cdot g \cdot c_{r} \cdot \cos \left(\alpha_{s l}(t)\right)
$$

When the vehicle is running through a curve, the rolling resistance increases. This additional curve resistance force is modeled by the subsequent equation [17]:

$$
F_{r c}=\left(m_{v}+m_{l}\right) \cdot g \cdot c_{r c}\left(v, r_{c}(t)\right)
$$

The curve resistance coefficient $c_{r c}$ and thus the curve resistance force $F_{r c}$ depend on the lateral acceleration $a_{l}=\frac{v^{2}}{r_{c}}$ acting on the vehicle, where $r_{c}$ specifies the curve radius. Fig. 3 shows a non-linear dependency for this relation. However, the figure covers extremely high lateral accelerations that do not occur in safe and comfortable driving states. Hence, the lateral acceleration is limited to $2.5 \frac{\mathrm{m}}{\mathrm{s}^{2}}$ and the curve in Fig. 3 is linearised for the interval $\left[0 \frac{\mathrm{m}}{\mathrm{s}^{2}}, 2.5 \frac{\mathrm{m}}{\mathrm{s}^{2}}\right]$. With the linearised curve resistance coefficient $c_{r c}^{*}$ the following relation for the curve resistance force is obtained:

$$
F_{r c} \approx\left(m_{v}+m_{l}\right) \cdot g \cdot c_{r c}^{*} \cdot \frac{v(t)^{2}}{r_{c}(t)}
$$

The grade resistance force $F_{g r}$ depends on the road slope angle as well [16].

$$
F_{g r}=\left(m_{v}+m_{l}\right) \cdot g \cdot \sin \left(\alpha_{s l}(t)\right)
$$

The air drag resistance force $F_{d}$ is a function of the square of the driving speed $v$. The coefficients are related to the shape of the vehicle (projected front surface area $A_{v}$, air drag coefficient $c_{d}$ and the air density $\rho_{a}$ ) [16].

$$
F_{d}=\frac{1}{2} \cdot \rho_{a} \cdot c_{d} \cdot A_{v} \cdot v(t)^{2}
$$

Given these forces, the acceleration of the vehicle in longitudinal direction can be computed from the difference between the traction force and the driving resistance forces divided by the inert mass of the vehicle. Tyre slip is disregarded here since a non-aggressive driving style is intended.

$$
\begin{aligned}
\frac{d v(t)}{d t}= & \left(F_{\text {trac }}(t)-F_{r}\left(\alpha_{s l}(t)\right)-F_{r c}\left(r_{c}(t), v(t)\right)\right. \\
& \left.-F_{g r}\left(\alpha_{s l}(t)\right)-F_{d}(v(t))\right) / m_{e q}
\end{aligned}
$$

The equivalent mass $m_{e q}$ is given by the relation $m_{e q}=\left(m_{v}+m_{l}\right) \cdot e_{i}$ which takes into account the rotational inertia of the drive train components by augmenting the vehicle mass $\left(m_{v}+m_{l}\right)$ by the constant factor $e_{i}$.

The vehicle specific parameters in (1) to (6) are accessible from data sheets of the Smart ED [18]. The rolling resistance coefficient $c_{r}$ is assumed to be 0.013 . The gravitational acceleration is assumed to be $g=9.81 \frac{\mathrm{m}}{\mathrm{s}^{2}}$ and the density of surrounding air to be $\rho_{a}=1.2 \frac{\mathrm{kg}}{\mathrm{m}^{3}}$. All parameters of the model describing the experimental car are summarised in Table I.

Table I. Parameters of the motion model of the Smart ED.

\begin{tabular}{llcc}
\hline symbol & value & symbol & value \\
\hline$A_{v}$ & $1.95 \mathrm{~m}^{2}$ & $m_{e q}$ & $1070 \mathrm{~kg}$ \\
$c_{d}$ & 0.37 & $m_{l}$ & $160 \mathrm{~kg}$ \\
$c_{r}$ & 0.01 & $m_{v}$ & $900 \mathrm{~kg}$ \\
$g$ & $9.81 \mathrm{~m} / \mathrm{s}^{2}$ & $\rho_{a}$ & $1.2 \mathrm{~kg} / \mathrm{m}^{3}$ \\
$e_{i}$ & 1.01 & & \\
\hline
\end{tabular}




\subsection{Model of the charge consumption}

In order to relate the electrical input of the drive train with the mechanical power output, the drive train and motor characteristics must be modeled. The motor of the test vehicle is an AC machine that is supplied by the DC battery via power electronics. A detailed model of all physical processes in these components is not suitable for the simulation of a cruise control loop due to its complexity.

Since the goal of the controller is to minimise the charge consumption $C_{e l}=\int_{t_{0}}^{t_{\text {end }}} i_{\text {batt }} \cdot d t$ of the car, the terminal battery current $i_{\text {batt }}$ has been measured on a dynamometer test bench in different static operating points (specified by the driving speed $v$ and traction force $F_{\text {trac }}$. These measurements are then summarised in a lookup table to model the charge consumption of the vehicle. The battery state of charge has been kept between 70 and $100 \%$ throughout the measurements to avoid the influence of a limited battery state of charge.

The resulting interpolated characteristics are depicted in Fig. 4 and are included in the dynamic model to establish the link between mechanical and electrical characteristics. Static characteristic maps are widely used in drivetrain modeling [16]. It can be assumed that the characteristics measured in quasi-static operation also hold in dynamic operation since the dynamics of the vehicle (driving speed and battery state of charge) are much slower than the dynamics related to the electrical components (battery current, motor current and torque).

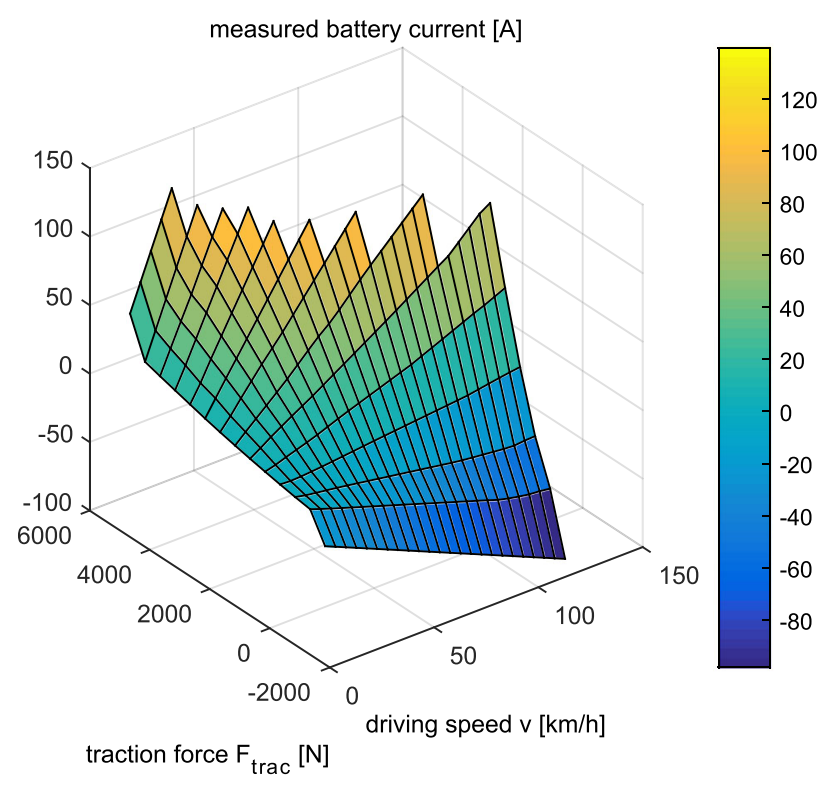

Fig. 4. Terminal battery current of the Smart ED as a function of driving speed and traction force.
It should be noted that the influence of all drivetrain components (battery, power electronics, motor, transmission, wheels) on the charge consumption is included in the measured data for high states of charges. However, low battery charges can change the consumption characteristics. Nevertheless, since a controller prediction model is the design goal here, the characteristics can be assumed to be constant throughout one prediction horizon. For different states of charge, different characteristics can be included in the model.

\section{CONTROLLER DESIGN}

This section describes the synthesis of the energy-efficient model-predictive cruise controller for an electric vehicle. Several transformations and reformulations are presented to bring the dynamic models of Section 3.2 into a suitable form without applying an overall linearisation.

\subsection{Dynamic motion model for the controller design}

The basis for the motion equation is (6). For the application of a predictive cruise controller, it is useful to describe the model as a function of the position instead of time since the inputs related to the road ahead (slope angle, curvature and speed limits) are also given as functions of the position. The model can be reformulated by applying the following transformation:

$$
\frac{d}{d s}=\frac{d}{d t} \cdot \frac{d t}{d s}=\frac{d}{d t} \cdot \frac{1}{v}
$$

The reformulation (7) consequently leads to a motion equation depending on the inverse of a state variable (the velocity $v$ ). This is disadvantageous for a fast solution of the optimisation problem. Inspired by an idea in related works $[19,20]$, a second reformulation is applied to calculate the kinetic energy

$$
e_{k i n}=\frac{1}{2} \cdot m_{e q} \cdot v(t)^{2}
$$

of the moving vehicle instead of the driving speed. Since only positive speed values are considered, the speed can be calculated from the kinetic energy values at a given vehicle mass after the optimisation. Derivation of (8) with respect to position $s$ yields

$$
\frac{d e_{k i n}}{d s}=m_{e q} \cdot \frac{d v}{d t}
$$

By applying (9) to the motion equation (6), the following linear differential equation is obtained. The values of the sine and cosine functions of the slope angle $\alpha_{s l}(s)$ are 
considered as measurable disturbances and assumed to be known. The curve radius $r_{c}(s)$ is assumed to be a given parameter of the model. In reality, it can be extracted from digital maps.

$$
\begin{aligned}
\frac{d e_{k i n}}{d s}= & F_{t r a c}(s)-F_{r}\left(\alpha_{s l}(s)\right)-F_{r c}\left(r_{c}(s), e_{k i n}(s)\right) \\
& -F_{g r}\left(\alpha_{s l}(s)\right)-F_{d}\left(e_{k i n}(s)\right)
\end{aligned}
$$

Herein, $F_{r c}$ and $F_{d}$ are rewritten in terms of kinetic energy:

$$
\begin{aligned}
& F_{r c}\left(e_{k i n}\right)=\frac{2 \cdot\left(m_{v}+m_{l}\right) \cdot g}{m_{e q} \cdot r_{c}(s)} \cdot c_{r c}^{*} \cdot e_{k i n}(s) \\
& F_{d}\left(e_{k i n}\right)=\frac{1}{m_{e q}} \cdot \rho_{a} \cdot c_{d} \cdot A_{v} \cdot e_{k i n}(s)
\end{aligned}
$$

Note, that the curve radius in (11) depends on the position $s$ which leads to a position-variant model.

Since (10) is only valid for positive kinetic energy values, the inequality constraint

$$
e_{k i n} \geq 0
$$

must be added to the optimisation problem.

To stay within the limitations of the vehicle, the traction force needs to be limited. The measured full-load curve as well as the traction force at coasting (giving the maximum and minimum possible traction force depending on the kinetic energy of the moving vehicle) of the Smart ED are given in Fig. 5. The full-load curve has been measured at fully pushing the accelerator pedal but without pressing the "kick-down" switch below the accelerator pedal. The traction force at coasting has been measured with released pedals. It should be noted that the vehicle recovers electrical energy if the accelerator pedal is released (resulting in negative traction forces). By this, slight regenerative braking is possible without actuating the brake pedal.

Since only linear constraints should be considered here, the measured curves are linearised using a least-squares approximation, resulting in the hatched polygon in Fig. 5 and represented by the following linear inequality:

$$
g_{1} \cdot e_{k i n}+g_{2} \leq F_{\text {trac }} \leq g_{3} \cdot e_{k i n}+g_{4} ; \quad e_{k i n} \geq 0
$$

In the region of medium kinetic energies where the linear constraint allows higher traction forces than the measured full-load curve, it is possible that the traction force planned by the MPC is above maximum realisable traction force in the car. This is considered later in the closed-loop simulation by saturating the output of

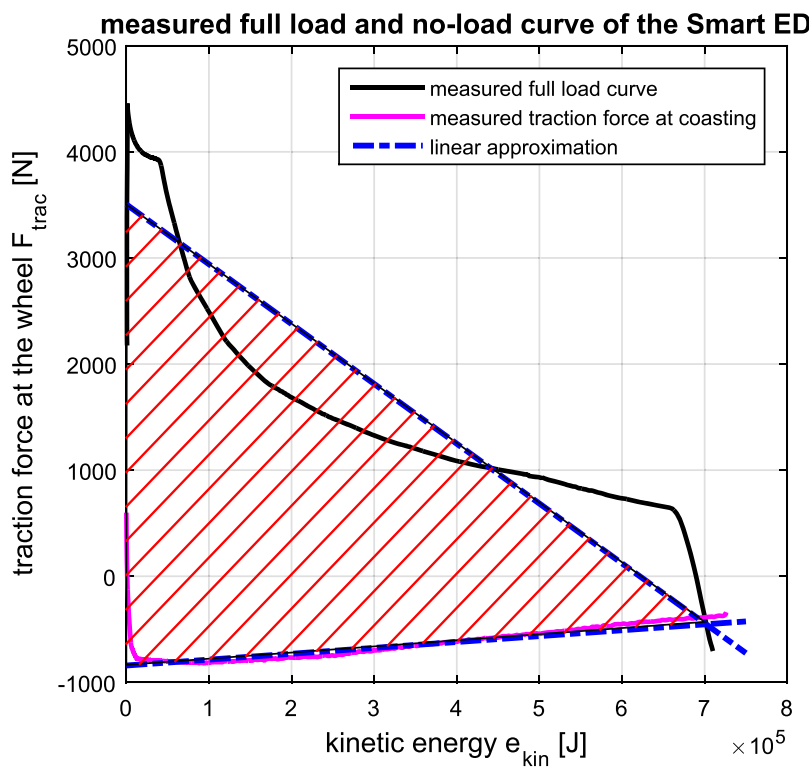

Fig. 5. Measured traction force of the Smart ED at full-load (100\% accelerator position, black line) and coasting (pedals released, pink line). The linear approximation is given in blue. The hatched area is the feasible region of the traction force $F_{\text {trac }}$.

the controller according to the measured full-load curve to represent the behaviour of the real car. The effect of this saturation might lead to a mismatch between the predicted and the actual vehicle behaviour but can be compensated by the controller.

\subsection{Charge consumption model for the controller design}

The vehicle motion model (10) is formulated with respect to the position $s$. Hence, the charge consumption must also be derived in terms of position. Here, it is advantageous that every operating point in the current consumption map (Fig. 4) is related to a certain driving speed due to the fixed transmission ratio. Thus, each point of the current consumption map is divided by its related driving speed $v$ according to (7) to obtain the charge consumption per meter. In addition to this, the $\mathrm{x}$-axis is rescaled in terms of the kinetic energy of the moving vehicle in order to fully comply with the reformulated motion equation (10). The resulting map of the charge consumption per meter as a function of the kinetic energy and the traction force is given for the Smart ED in Fig. 6a. The objective is to implement an approximation of these characteristics in the underlying dynamic model of the controller.

A common method for the approximation of measured data is the use of polynomials [16,21]. Nevertheless, 

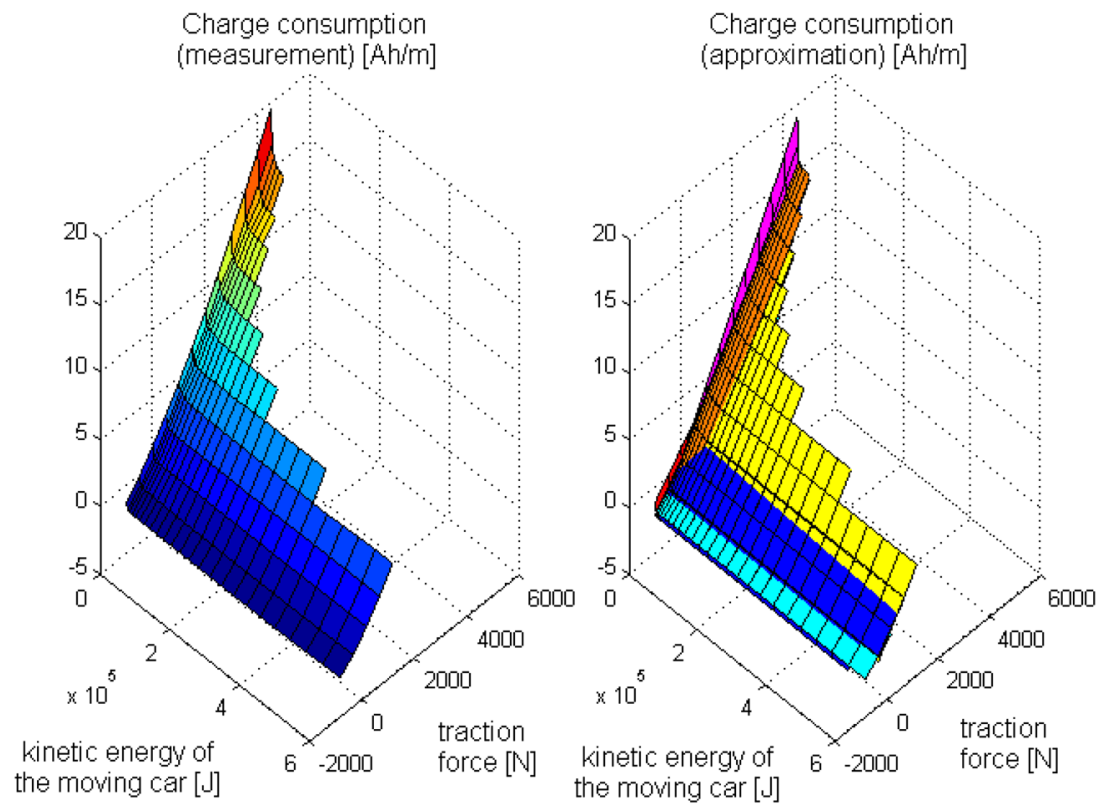

Fig. 6. The figure shows the vehicle charge consumption per meter. The x-axis has been rescaled in terms of the kinetic energy of the moving vehicle. a) gives the measured characteristics; b) gives the approximation by six linear inequalities.

a closer look at Fig. 6a shows that the given characteristics are more suitable for a piecewise linear approximation since they can hardly be captured by one single lower order polynomial that is suitable for the use in a real-time optimisation. The use of a piecewise linear problem formulation would be appropriate but in general requires the use of different dynamic models in different regions of the state space (i.e. operating points) which makes the optimisation problem more time-consuming to solve and less suitable for a real-time implementation. This can be avoided by using a convex piecewise linear approximation. Convex piecewise linear functions can be represented by the maximum of a set of linear functions. This maximum can be determined implicitly in the optimisation problem with no need for a special solver.

Approximating non-linear maps by (the maximum of) linear functions is a known technique in non-linear optimisation and is called separable programming [22]. Finding a convex piecewise linear approximation with a limited number of functions results in the minimisation of the cardinality of a set of functions and leads to a (hard) combinatorial optimisation [23]. Thus, a heuristic iterative algorithm such as presented in [24] is used here to find a convex piecewise linear model of the charge consumption, see the following procedure.

1. Start with a set of data points $Y$ defined at the grid points $U$ and an initial set of $K$ approximating linear functions $\mathcal{P}_{\text {fun }}=\left\{P_{1} \ldots P_{K}\right\} ; P_{i}=a_{i} \cdot e_{k i n}+b_{i}$. $F_{\text {trac }}+c_{i}$, represented by the coefficients $a_{i}, b_{i}$ and $c_{i}$.
2. Determine the subsets of data grid points $U_{i}$ where each of the linear functions $P_{i}$ yields the maximum function value of the set $\mathcal{P}_{\text {fun }}$ and assign these grid points and the associated data points $Y_{i}$ to that function.

3. Update the coefficients of the linear functions by least-squares fitting of the functions to those data points that have been assigned to it in the previous step: $\min _{a_{i}, b_{i}, c_{i}} \sum\left(P_{i}\left(U_{i}\right)-Y_{i}\right)^{2}$

4. If the updated coefficients are the same as those of the previous iteration, return $a_{i}, b_{i}$ and $c_{i}$ as the result of the converged algorithm (i. e. the linear are functions are fitted to those data points, where they represent the maximum of the set of functions $\left.\mathcal{P}_{\text {fun }}\right)$.

5. Else: go to step 2.

If the algorithm converges, it delivers a set of linear functions that are fitted to those regions of the data grid where they represent the maximum of $\mathcal{P}_{\text {fun }}$ and thus where they become relevant for the approximation. We refer to [24] for further details on this algorithm.

Here, the application of six linear functions has shown a good compromise between fitting error and computational complexity. A higher number of planes does not yield a significant improvement in accuracy. For six linear functions $\left(P_{1}\right.$ to $\left.P_{6}\right)$, the piecewise linear fit in Fig. $6 \mathrm{~b}$ is obtained with this method. 
$P_{1}$ to $P_{6}$ are included as inequality constraints on a decision variable $u_{\text {cons }}$ that represents the charge consumption of the vehicle per meter and have the form:

$$
P_{i}: \quad u_{\text {cons }} \geq a_{i} \cdot e_{\text {kin }}+b_{i} \cdot F_{\text {trac }}+c_{i} \quad \mathrm{i}=1 \ldots 6
$$

The optimisation problem of the MPC controller (as discussed later in Section 4.4) is then set up in a way that it determines the maximum of the constraint functions $P_{1}$ to $P_{6}$ implicitly and guarantees that the decision variable $u_{\text {cons }}$ represents the charge consumption per meter of the vehicle according to the following piecewise linear model:

$$
\begin{array}{ll}
u_{\text {cons }}=a_{i} \cdot e_{k i n}+b_{i} \cdot F_{\text {trac }}+c_{i} & \text { if } P_{i} \text { is active } \\
u_{\text {cons }} \geq a_{j} \cdot e_{\text {kin }}+b_{j} \cdot F_{\text {trac }}+c_{i} & \text { for } j \neq i
\end{array}
$$

Herein, it becomes obvious that one of the inequality constraints (15) is always active depending on the actual operating point (specified by the speed $v$ and the traction force $F_{\text {trac }}$ ).

Given this information, the charge consumption $C_{e l}$ of the vehicle can simply be modeled by integrating the decision variable $u_{\text {cons }}$ (representing the charge consumption per meter) with respect to the position.

$$
\frac{d C_{e l}}{d s}=u_{c o n s}
$$

\subsection{Model discretisation}

The continuous dynamic motion model (10) and the model of the charge consumption (17) can be summarised in state space form and discretised straightforwardly by assuming zero-order hold for the input and the disturbance. The following discrete linear state space representation (with the position-variant system matrix $A_{d, k}$ ) is obtained for $k \in\{0 \ldots . N-1\}$, where " "symbolises the discrete counterparts of the continuous variables:

$$
\begin{array}{r}
\underbrace{\left[\begin{array}{c}
\hat{e}_{k i n, k+1} \\
\hat{C}_{e l, k+1}
\end{array}\right]}_{\hat{x}_{k+1}}=\underbrace{\left[\begin{array}{rr}
a_{11, k} & 0 \\
0 & 1
\end{array}\right]}_{A_{d, k}} \cdot \underbrace{\left[\begin{array}{c}
\hat{e}_{k i n, k} \\
\hat{C}_{e l, k}
\end{array}\right]}_{\hat{x}_{k}} \\
+\underbrace{\left[\begin{array}{cc}
b_{11} & 0 \\
0 & b_{22}
\end{array}\right]}_{B_{d}} \cdot \underbrace{\left[\begin{array}{c}
\hat{F}_{\text {trac }, k} \\
\hat{u}_{c o n s, k}
\end{array}\right]}_{\hat{u}_{k}}+\underbrace{\left[\begin{array}{cc}
e_{11} & e_{12} \\
0 & 0
\end{array}\right]}_{E_{d}} \cdot \underbrace{\left[\begin{array}{c}
\sin \left(\hat{\alpha}_{s l, k}\right) \\
\cos \left(\hat{\alpha}_{s l, k}\right)
\end{array}\right]}_{\hat{d}_{k}}
\end{array}
$$

\subsection{Overall problem formulation}

Based on the results of the previous sections, the complete model-predictive eco-cruise control problem is formulated as a discrete-position finite-dimensional optimisation problem with a quadratic cost function and linear constraints. The cost function includes the charge consumption at the last step of the prediction horizon to achieve an "intelligent" predictive controller behaviour with the freedom to increase the consumption at any position if there is the benefit to save more energy later as a result of this anticipatory action:

$$
M_{1}=q_{1} \cdot \hat{C}_{e l, N}=q_{1} \cdot \sum_{k=0}^{N-1} \hat{u}_{c o n s, k} \cdot \Delta s
$$

The kinetic energy tracking error at the last step of the prediction horizon is included in the cost function to enable closed-loop stability for the system state kinetic energy:

$$
\left.M_{2}=q_{2} \cdot\left(\hat{e}_{k i n, N}-\hat{e}_{k i n, r e f, N}\right)\right)^{2}
$$

Finally, the accumulated squared kinetic energy tracking error is a measure for the deviation from the speed reference trajectory

$$
L_{1}=q_{3} \cdot \sum_{k=1}^{N}\left(\hat{e}_{k i n, k}-\hat{e}_{k i n, r e f, k}\right)^{2}
$$

and the accumulated squared deviation of the control input traction force from the reference is minimised to make the control commands smoother and stable. $\hat{F}_{\text {trac,ref }}$ is the traction force necessary to drive constantly at the reference speed and can be computed from the sum of the driving resistance forces.

$$
L_{2}=q_{4} \cdot \sum_{k=0}^{N-1}\left(\hat{F}_{\text {trac }, k}-\hat{F}_{\text {trac, } r e f, k}\right)^{2}
$$

The complete optimisation problem is given as follows:

$$
\min _{\hat{F}_{\text {trac }}, \hat{u}_{\text {cons }}} M_{1}+M_{2}+L_{1}+L_{2}
$$

subject to:

$$
\begin{aligned}
& \hat{x}_{k+1}=A_{d, k} \cdot \hat{x}_{k}+B_{d} \cdot \hat{u}_{k}+E_{d} \cdot \hat{d}_{k} \\
& \hat{C}_{e l, 0}=C_{e l}(0) ; \quad \hat{e}_{k i n, 0}=e_{k i n}(0) \\
& \quad 0 \leq \hat{e}_{k i n, k} \\
& g_{1} \cdot \hat{e}_{k i n, k}+g_{2} \leq \hat{F}_{\text {trac }, k} \leq g_{3} \cdot \hat{e}_{k i n, k}+g_{4} \\
& \hat{u}_{\text {cons }, k} \geq a_{i} \cdot \hat{e}_{k i n, k}+b_{i} \cdot \hat{F}_{\text {trac }, k}+c_{i} \quad \mathrm{i}=1 \ldots 6
\end{aligned}
$$

The reference tracking versus energy saving performance can be tuned by adjusting the weighting factors $q_{1}$ and $q_{3}$ in the cost function of the optimal control 
problem (22). By increasing the value of $q_{3}$ and/or lowering the value of $q_{1}$, the reference tracking can be improved. However, in the context of eco-cruise control, it is desired to allow deviations from the reference in order to use this freedom to save electrical energy.

As already mentioned in Section 3.2, the problem formulation (23) includes a piecewise linear model of the decision variable $\hat{u}_{\text {cons }}$ specified by the inequality constraints (23e). In the cost function, $\hat{u}_{\text {cons }}$ only affects the charge consumption $\hat{C}_{e l}$ (see (17), (19)), i.e. the cost function is separable with regard to this variable. Making the value of $\hat{C}_{e l, N}$ as small as possible can only be achieved by making the decision variable $\hat{u}_{\text {cons }}$ as small as possible at each position step. Hence, $\hat{u}_{\text {cons }}$ will always lie on the boundary of the feasible region defined by the inequality constraints (23e). Since different constraints become active in different operating points, this leads to a problem formulation that is equivalent to the use of a piecewise linear model of the charge consumption per meter $\hat{u}_{\text {cons }}$ without the necessity of using a special solver.

Since problem (23) only consists of linear and quadratic cost function terms as well as linear constraints, it can be rewritten in the standard form of quadratic programming with the optimisation variables $y$ and the weighting matrices $Q_{w}$ and $h_{w}$ :

$$
\min _{y} \frac{1}{2} \cdot y^{T} Q_{w} y+h_{w}^{T} \cdot y \quad \text { s.t. } \quad A_{c o n} \cdot y \leq b_{c o n}
$$

If $Q_{w}$ in problem (24) is positive definite, the quadratic program is strictly convex. The transformation of problem (23) into the form of (24) and the proof of convexity are omitted here for brevity.

\subsection{Closed-loop stability}

Since the MPC problem is converted to a linear MPC formulation, the classical approach to establish closed-loop stability by introducing a terminal weight on the states that approximates the infinite horizon cost can be applied according to $[25,26]$. However, some adaptations are necessary in the given case. The classical MPC theory assumes all states of the system to be steadily regulated to a constant set-point. In the application of a charge-saving cruise controller, driving at a constant speed is considered as a stable system state while the state charge consumption $\hat{C}_{e l}$ can increase steadily and cannot be driven to a constant set-point. Thus, only the state kinetic energy of the moving car $\hat{e}_{k i n}$ is considered in the stability analysis while the energy consumption is disregarded.

Further, the reference and disturbance trajectory must be assumed to be constant beyond the prediction horizon to enable an infinite horizon equivalent controller. Then, to make the control formulation equivalent to an infinite horizon approach [26], the weight $q_{2}$ on the terminal kinetic squared energy tracking error can be computed as the limit of the series $\bar{Q}=\sum_{i=0}^{\infty}\left(A_{d}^{T}\right)^{i} Q A_{d}^{i}$ where $Q=\left[\begin{array}{cc}q_{3} & 0 \\ 0 & 0\end{array}\right]$ and $\bar{Q}=\left[\begin{array}{cc}q_{2} & 0 \\ 0 & 0\end{array}\right]$. This results in the value $q_{2}=12.56$.

In addition, the prediction horizon needs to be sufficiently long and a positive weight $q_{4}$ must be imposed on the accumulated deviation of the control input traction force from the value necessary to drive steadily at the reference to ensure theoretical closed-loop stability under the given assumptions [26].

\section{SPEED REFERENCE GENERATION}

A predictive cruise controller cannot be purely based on the minimisation of the charge consumption. The control input must be determined in accordance with topological information about the road (curvature, inclination), legal aspects (speed limits) as well as the traffic situation (distance to the preceding car). The controller design offers the freedom to include these influences either in the constraints or in the cost function of the underlying optimisation problem.

Including speed constraints can easily lead to an infeasible optimisation problem if the constraints cannot be fulfilled. For this reason, the speed limits, the road topology and the distance to the preceding car are considered here in the speed reference trajectory.

The reference trajectory $v_{r e f}(s)$ is defined as the minimum of the admissible speeds resulting from the speed limits $v_{\text {lim }}(s)$, the road curvature $v_{\text {cur }}(s)$ and the following of the preceding car $v_{h}(s)$. In the sequel, $v_{\text {lim }}(s), v_{\text {cur }}(s)$ and $v_{h}(s)$ are defined.

$$
v_{\text {ref }}(s)=\min \left\{v_{\text {lim }}(s), v_{\text {cur }}(s), v_{h}(s)\right\}
$$

The admissible speed resulting from speed limits $v_{\text {lim }}(s)$ is directly the active speed limit at the related vehicle position $s$. The assumed relation between the curve radius $r_{c}$ and the desired curve speed $v_{c u r}(s)$ used in this work is given in Fig. 7.

While the speed limits and the road topology are given from the digital maps before the trip, the traffic situation changes and requires an update of the speed reference for every sample step. If a preceding car is detected, several cases are distinguished. The speed of the preceding car $v_{p}$ (assumed to be given and constant throughout the prediction horizon) could be higher or lower than the speed of the host vehicle $v_{h}$ and the 


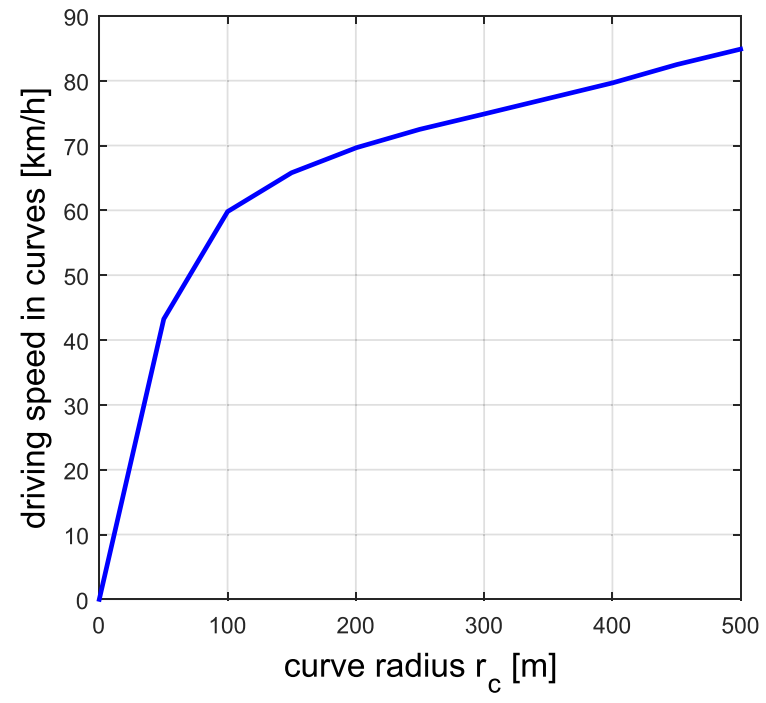

Fig. 7. Assumed relation between the curve radius and the desired curve speed. measured clearance $d_{h, 0}$ could be above or below the safety distance $d_{h, \text { safe }}$. The safety distance is here defined according to the speed of the preceding vehicle to be $d_{h, s a f e}[m]=\frac{1}{2} \cdot v_{p}[\mathrm{~km} / \mathrm{h}]$.

If the actual distance is above the safety distance $\left(d_{h, 0}>d_{h, \text { safe }}\right)$ and the speed of the preceding car is above the host vehicle speed $\left(v_{p} \geq v_{h, 0}\right)$, there is no influence of the preceding car on the host vehicle speed considered.

If the preceding car is slower than the host vehicle $\left(v_{p}<v_{h, 0}\right)$ and the distance is above the safety distance, i.e. $v_{p}<v_{h, 0}$ and $d_{h, 0}>d_{h, \text { safe }}$, a safe but also smooth approach to the speed of the preceding car $\left(v_{h} \rightarrow v_{p}\right)$ and the safety distance $\left(d_{h} \rightarrow d_{h, \text { safe }}\right)$ is required. In order to obtain a strong decrease of the speed at the beginning of the prediction horizon (to be safe) and then subsequently a smooth approach of the speed of the preceding vehicle, an exponential decay function beginning at $v=v_{h, 0}$ for $s=0$ and approaching $v=v_{p}$ for $s \rightarrow \infty$ is defined, see Fig. 8 (dash-dotted blue curves).

$$
v_{h}=v_{p}+\left(v_{h, 0}-v_{p}\right) \cdot e^{-a_{d e c} \cdot s}
$$
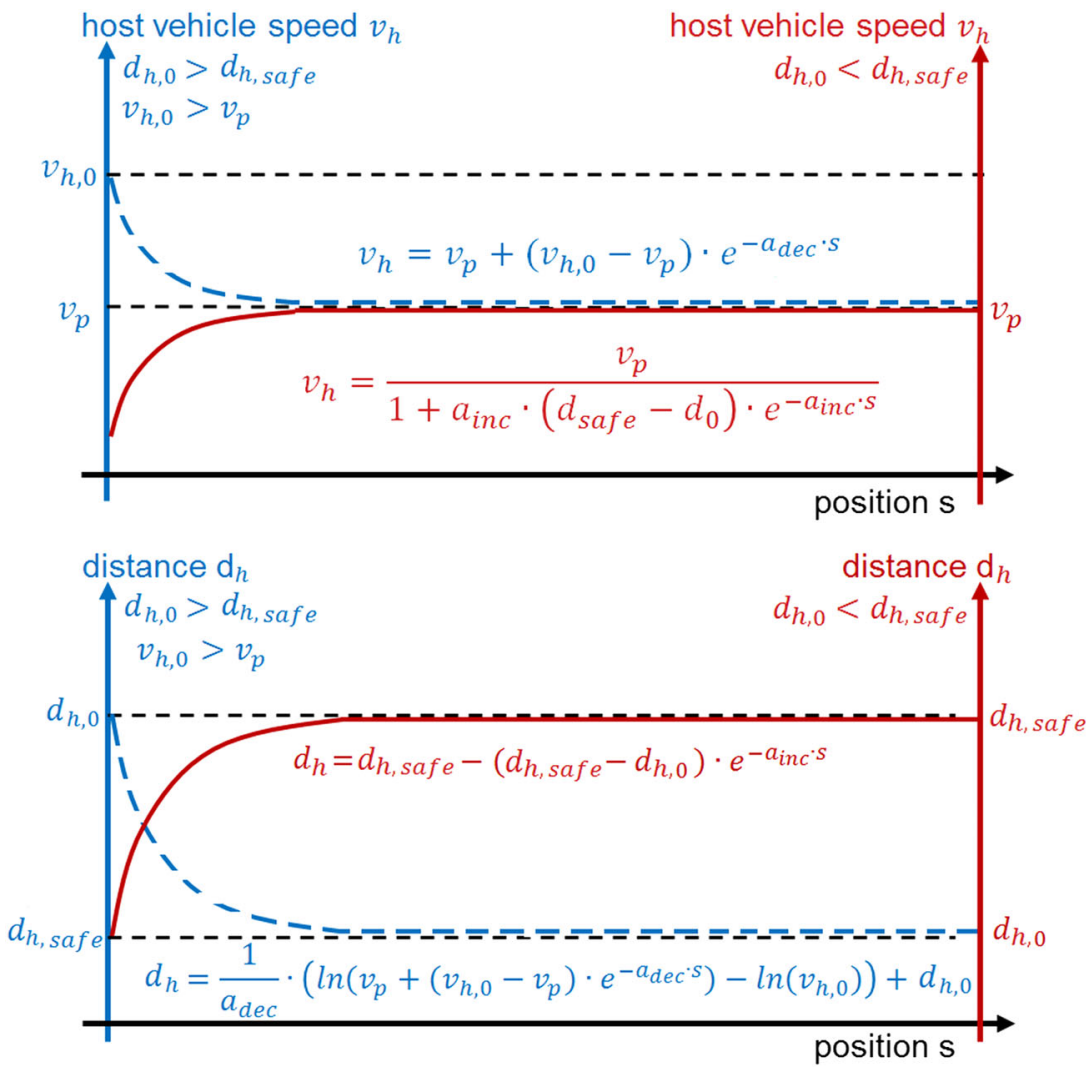

Fig. 8. The figure shows the speed reference depending on the distance to the preceding car. The blue (dash-dotted) case shows the approach of a slower preceding car while the red (continuous) case shows the case that the clearance between the cars must be increased to reach the safety distance $d_{h, s a f e}$. 
The parameter $a_{d e c}$ determines the rate of the exponential decay and is determined such that the safety distance $d_{h, s a f e}$ is reached just when the speed of the host vehicle $v_{h}$ almost reaches the speed of the preceding car $\left(v_{h}=1.01 \cdot v_{p}\right)$ which results in following the preceding car with the same speed at the safety distance. To derive an equation for $a_{d e c}$ accordingly, (26) is inserted into the formula of the derivate of the distance between the two cars with respect to the host vehicle position:

$$
\frac{d d_{h}}{d s}=\frac{v_{p}-v_{h}}{v_{h}}
$$

Inserting (26) into (27) and integration with respect to $s$ yields the relation for the distance between two cars throughout the prediction horizon:

$$
\begin{gathered}
d_{h}(s)=\frac{1}{a_{d e c}} \cdot \ln (\underbrace{v_{p}+\left(v_{h, 0}-v_{p}\right) \cdot e^{-a_{d e c} \cdot s}}_{v_{h}}) \\
\underbrace{-\frac{1}{a_{d e c}} \ln \left(v_{h, 0}\right)+d_{0}}_{\text {integration const. }}
\end{gathered}
$$

Demanding $d_{h}:=d_{h, \text { safe }}$ and $v_{h}:=1.01 \cdot v_{p}$, yields the required relation to determine $a_{d e c}$ such that the safety distance is reached just when the cars run with almost the same speed:

$$
a_{\text {dec }}=\frac{\ln \left(1.01 \cdot v_{p}\right)-\ln \left(v_{h, 0}\right)}{d_{h, \text { safe }}-d_{h, 0}}
$$

In the case that the actual distance is below the safety distance $\left(d_{h, 0}<d_{h, \text { safe }}\right)$, an exponential increase of the distance $d_{h, 0} \rightarrow d_{h, \text { safe }}$ is demanded according to (see Fig. 8, continuous red curves):

$$
d_{h}=d_{h, \text { safe }}-\left(d_{h, \text { safe }}-d_{h, 0}\right) \cdot e^{-a_{\text {inc }} \cdot s}
$$

With the help of (27), the resulting speed profile can be derived:

$$
v_{h}=\frac{v_{p}}{1+a_{i n c} \cdot\left(d_{h, s a f e}-d_{h, 0}\right) \cdot e^{-a_{i n c} \cdot s}}
$$

$a_{i n c}$ is tuned with a simple heuristic relation according to $d_{h, \text { safe }}$ as follows: $a_{\text {inc }}=1 / d_{h, \text { safe }}$.

In the case that $0.95 \cdot v_{p} \leq v_{h, 0} \leq 1.05 \cdot v_{p}$ and $0.95 \cdot d_{h, \text { safe }} \leq d_{h, 0} \leq 1.05 \cdot d_{h, \text { safe }}$, the speed reference is constantly set to $v_{h}=v_{p}$. The interval is chosen to avoid oscillations between the different modes (26) and (31). The equations and speed profiles related to the car following behaviour are summarised in Fig. 8.
To comply with the internal controller model, the speed reference trajectory is converted into a kinetic energy reference $e_{\text {kin,ref }}(s)$ using (8).

\section{SIMULATION OF THE CLOSED-LOOP CONTROL}

While all dynamic models are identified from the real Smart ED, the controller behaviours shown and assessed in the following are first obtained in a simulation for safety reasons.

The simulation of the control loop is performed with the non-linear plant model described in Section III and the controller designed in Section IV. The scenario includes a sequence of a speed limit change, driving through a curve, down-slope and up-slope driving as well as a suddenly appearing preceding car according to Fig. 9. The prediction horizon of the MPC controller is subdivided into 40 steps of $10 \mathrm{~m}$. The scenario is simulated three times with the proposed MPC controller. First, with a zero weight on the charge consumption leading to pure kinetic energy reference tracking for comparison. Second, with adjusted weights for eco-driving and third, with a "soft" eco-driving approach using a lower weight on the charge consumption and a speed reference increased by $10 \%$. This "soft" eco-driving leads to the same average driving speed like the purely reference tracking controller to highlight that energy can be saved by changing the driving style without going slower.

The results of the above described simulation scenario are computed within MatLab/Simulink and are depicted in Fig. 9. The parameter values of the controller model are given in Table II while the simulation parameters are the same as in Section III (Table I).

The quadratic program (24) has been solved with the MatLaB function quadprog() and the solver interior-point-convex which has proven to work best in the simulations.

At first, only the eco-cruise controller and the purely reference tracking controller are compared. The purely kinetic energy reference tracking controller starts accelerating the car as fast as possible at the maximum traction force from standstill to the desired speed. The eco-cruise controller first accelerates fast but then reduces the traction force to avoid a high charge consumption. The further course of the results show the predictive behaviour of the eco-cruise controller. The simulated car decelerates already at the position of $670 \mathrm{~m}$ before the speed limit reduction to $50 \mathrm{~km} / \mathrm{h}$ in order the recover electrical energy. The subsequent acceleration starting at the position of $760 \mathrm{~m}$ is performed slowly such that high charge consumptions and especially braking in front of 

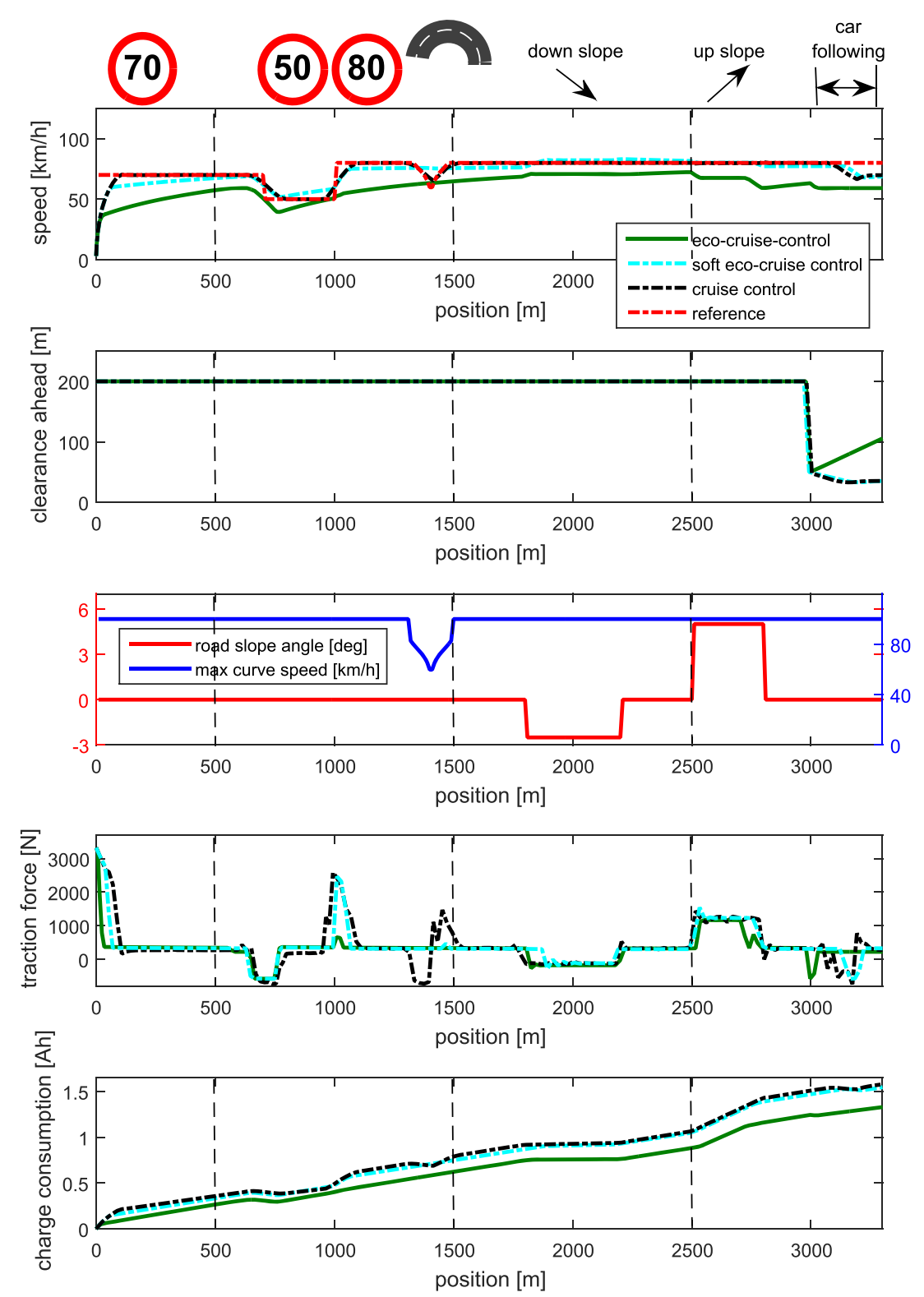

Fig. 9. Simulation results of the vehicle in closed-loop control. The green and cyan lines represent the results with the proposed eco-cruise controller while the black dashed lines result from pure speed-tracking MPC control.

the curve can be avoided. In comparison with the pure reference tracking MPC, the peaks in the traction force at the positions $1000 \mathrm{~m}$ and $1450 \mathrm{~m}$ can be completely avoided by the eco-cruise controller with the help of gradual accelerations.

At down-hill driving (position $1800 \mathrm{~m}$ to $2200 \mathrm{~m}$ ), the charge-saving vehicle slightly recovers electrical energy and accelerates at the same time. This kinetic energy reserve allows the vehicle to save charge on the following even road segment. In front of the up-slope, the driving speed is further increased from the position of $2310 \mathrm{~m}$ to gain momentum and thus reduce the traction force effort during the slope. During the up-hill driving ( $2500 \mathrm{~m}$ to $2800 \mathrm{~m}$ ), the traction must be increased but a lower driving speed is accepted to avoid a very high charge consumption.

A preceding car is assumed to be detected suddenly at the simulated position of $3000 \mathrm{~m}$ in a distance of $50 \mathrm{~m}$ and a speed of $70 \mathrm{~km} / \mathrm{h}$. While the purely reference-tracking controller slows down from $80 \mathrm{~km} / \mathrm{h}$ to $70 \mathrm{~km} / \mathrm{h}$ and keeps the safety distance of $35 \mathrm{~m}$, the eco-cruise controller decelerates below the speed of the preceding car and thus the clearance increases. 
To understand the behaviour of the eco-cruise controller, the simulated operating point trajectory is considered. Fig. 10 shows the projected top view on the piecewise linear approximated charge consumption map that has been presented in Fig. 6. Fig. 10 shows that the optimal trajectory mainly includes operating points at the intersection lines of the linear functions

Table II. Parameters of the eco-cruise controller setup.

\begin{tabular}{lccccc}
\hline Par. & Value & Par. & Value & Par. & Value \\
\hline$a_{1}$ & $-3.92 \mathrm{E}-4$ & $a_{2}$ & $-6.51 \mathrm{E}-6$ & $a_{3}$ & $2.71 \mathrm{E}-7$ \\
$a_{4}$ & $-9.93 \mathrm{E}-5$ & $a_{5}$ & $1.11 \mathrm{E}-7$ & $a_{6}$ & $9.14 \mathrm{E}-8$ \\
$b_{1}$ & 0.0040 & $b_{2}$ & 0.0033 & $b_{3}$ & 0.0033 \\
$b_{4}$ & 0.0025 & $b_{5}$ & 0.0018 & $b_{6}$ & 0.0027 \\
$c_{1}$ & 0.9620 & $c_{2}$ & 0.5808 & $c_{3}$ & 0.2880 \\
$c_{4}$ & 1.2918 & $c_{5}$ & 0 & $c_{6}$ & 0.5203 \\
$g_{1}$ & $5.538 \mathrm{e}-4$ & $g_{2}$ & -841.1 & $g_{3}$ & -0.0056 \\
$g_{4}$ & 3505 & $q_{1}$ & $1.26 \mathrm{e} 9$ & $q_{2}$ & 12.56 \\
$q_{3}$ & 0.25 & $q_{4}$ & 0.5 & $0.97 \leq a_{11, k} \leq 0.99$ \\
$b_{11}$ & 9.9595 & $e_{11}$ & $9.5218 \mathrm{e} 4$ & $e_{12}$ & 952.17 \\
$b_{22}$ & 10 & & & \multicolumn{3}{c}{} \\
\hline
\end{tabular}

approximating the charge consumption map to avoid entering inefficient regions of the characteristics. This explains the long segments of constant traction force in Fig. 9 and indicates that it is essential to choose the piecewise linear approximation of the charge consumption map properly.

The overall charge consumption in this simulation scenario is significantly reduced by the eco-cruise controller by $15.1 \%$ compared to the pure kinetic energy reference tracking predictive controller. At the same time, the average speed throughout the simulation is reduced by $16 \%$ The elapsed travel time with the purely reference tracking MPC is $167.7 \mathrm{~s}$ while the eco-cruise controller requires $200.7 \mathrm{~s}$ to reach the final position of $3300 \mathrm{~m}$. In a more realistic scenario with a lot of changes in the reference, even higher savings and a reduced loss of speed can be expected.

To show that energy savings are possible without reducing the average driving speed, the "soft" eco-cruise control is regarded where the weighting on the charge consumption has been lowered to $q_{1}=9 e 8$ and the

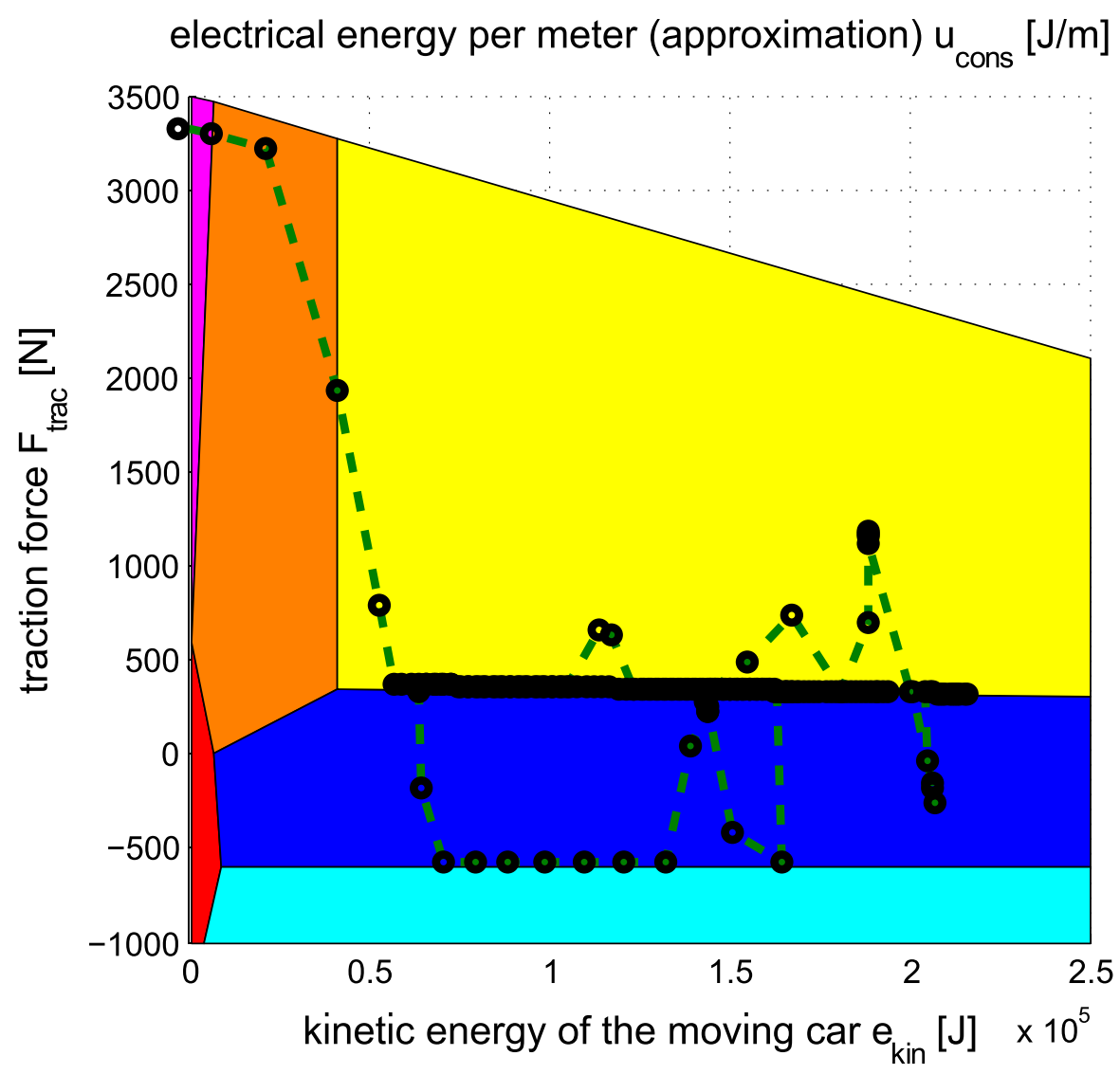

Fig. 10. Operating point trajectory (dashed green line) of the system controlled by the proposed eco-cruise controller. 

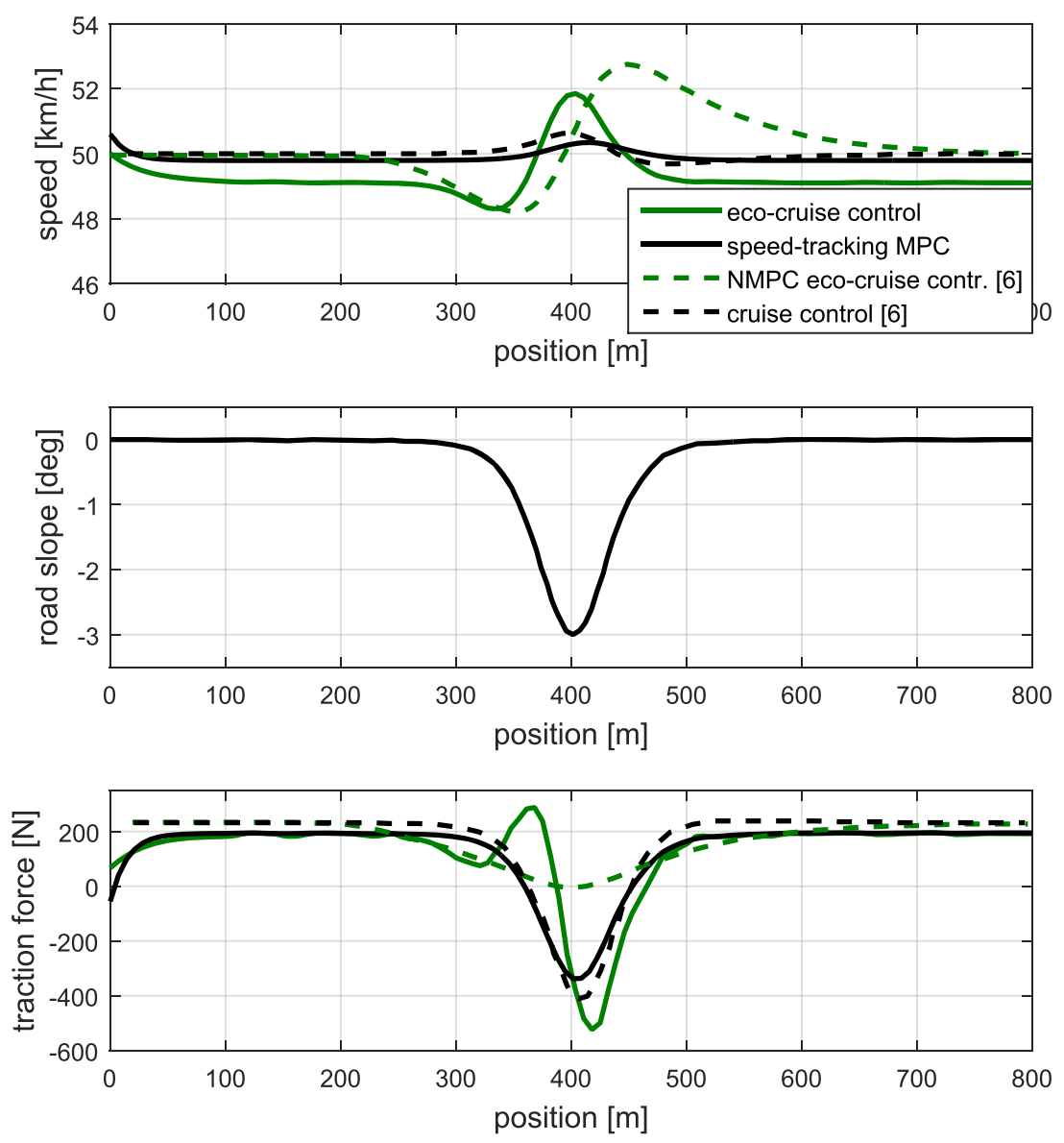

Fig. 11. Comparison of the control approach at hand to simulation results with a non-linear formulation presented in [6]; down-slope driving.

driving speed reference has been increased by $10 \%$. The results with this controller are plotted in cyan (dotted). The average driving speed of the purely reference tracking controller is maintained, here, while $2 \%$ of charge have been saved in comparison. The savings mainly come from gaining momentum throuout the down-slope and keeping the driving speed more constant.

In the experimental realisation, the optimisation is planned to be updated every 0.1 seconds. The discretisation step size of the prediction model is 10 metres. At the maximum driving speed of the Smart $E D(125 \mathrm{~km} / \mathrm{h})$, the time of $0.29 \mathrm{~s}$ passes to travel a distance of $10 \mathrm{~m}$. The control algorithm shall be executed significantly faster to update the control more frequently with optimisation results based on new measurements in order to react to changes in the traffic situation.

The computational time to solve the optimisation problem within MATLAB on a desktop PC (Intel Core i7) varies between 0.05 and 0.45 seconds during the presented simulation. In many cases, a real-time capable computation time is already achieved, here. Solving the same problem with a C-code based quadratic programming solver, a significantly faster computation can be expected. The problem dimension is defined by the number of optimisation variables (in this case 80) and the number of inequality constraints (in this case 360). [27] provides a study on the real-time capabilities of embedded quadratic programming using the $\mathrm{C}$-code generator $C V X G E N$. For a model-predictive control problem with large dimensions (336 optimisation variables, 252 inequality constraints), a computation time of $13 \mathrm{~ms}$ is achieved on a comparatively weak Intel Atom PC with a peak power consumption of $2 \mathrm{~W}$. The comparison of the presented model-predictive control approach in the work at hand to this reference allows the conclusion, that the proposed problem formulation is real-time capable. The controller is currently being implemented experimentally on a Linux-based embedded platform in the Smart ED to finally validate the results in real driving tests. 


\subsection{Comparison to a non-linear formulation}

This section compares the linear MPC approach at hand to the non-linear formulation given in [6] to compare the control reactions. Even though [6] presents an approach for fuel-powered cars, gear shifting is not considered in that work and thus the dynamic prediction model and energy-saving mechanisms are similar. The prediction horizon of the simulation examples in [6] is $10 \mathrm{~s}$ (the prediction model is formulated with respect to time) while the driving speed reference in the investigated scenarios is constant at $50 \mathrm{~km} / \mathrm{h}$. At this speed, the equivalent distance horizon is approximately $140 \mathrm{~m}$ which is used in this comparison to comply with the setup in [6]. Further, the weightings have been adjusted to $q_{1}=5 e 9, q_{2}=2216, q_{3}=45$ and $q_{4}=1.5 e 6$. Note that due to the adjustment of the prediction horizon and the weightings, the control reactions differ from the previous simulation presented in Fig. 9.

Figs. 11 and 12 shows the comparison of the simulation results. The green lines refer to the eco-cruise controllers while the black lines represent the purely reference tracking cruise controllers of the work at hand (continuous lines) and those presented in [6] (dash-dotted lines).

In the down-slope driving scenario, both eco-cruise control approaches show a deceleration followed by an acceleration. Throughout deceleration, energy can be saved while the momentum can be recovered again throughout the down-slope without traction force effort. This strategy is evident in both eco-cruise control approaches.

In the up-slope driving scenario, the eco-cruise controllers accelerate before reaching the up-slope section and decelerate during the slope in order to overcome the hill at a lower traction force and consequently a lower consumption.

In both scenarios, the proposed linear controller regulates the car to a driving speed below the reference in order to save energy due to the lower air drag. This behaviour is not given in the NMPC approach and results from different energy consumption models.
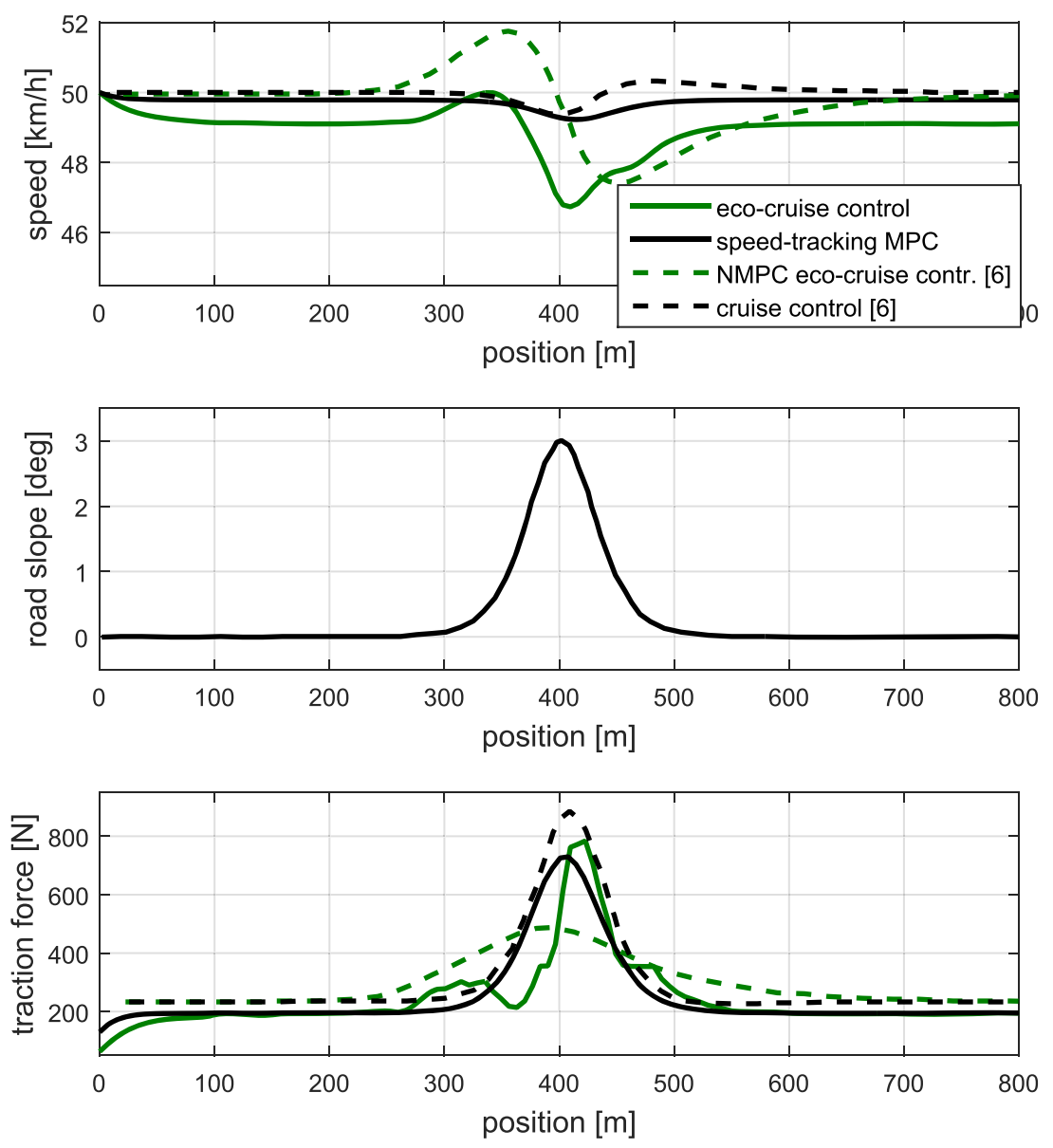

Fig. 12. Comparison of the control approach at hand to simulation results with a non-linear formulation presented in [6]; up-slope driving. 
In summary, it can be said that the linear approach at hand as well the non-linear method presented in [6] yield similar energy-saving mechanisms for up- and down-hill driving. This shows the effectiveness of the control formulation presented in this paper.

\section{CONCLUSION}

In the presented problem formulation, the ecocruise control problem is converted into a quadratic optimisation problem with linear constraints without applying an overall linearisation. The major nonlinearities are considered by using reformulations of the original problem. The proposed formulation guarantees a fast solution of the optimisation problem with guaranteed convergence and is much more suitable for a real-time implementation than a non-linear problem formulation. To complete the control approach, a speed reference generator is presented to provide an admissible reference trajectory based on road topology, speed limits and the traffic situation. As shown in Section VI, the proposed control system yields a significant potential to reduce the charge consumption of the electric vehicle.

\section{REFERENCES}

1. Barkenbus, J. N., "Eco-driving: An overlooked climate change initiative," Energy Policy, Vol. 38, No. 2, pp. $762-769$ (2010).

2. Rommerskirchen, C., M. Helmbrecht, and K. Bengler, "Increasing complexity of driving situations and its impact on an adas for anticipatory assistance for the reduction of fuel consumption," 2013 IEEE Intelligent Vehicles Symposium (iv), Gold Coast City, Australia, pp. 573-578 (2013).

3. Schwarzkopf, A. B. and R. B. Leipnik, "Control of highway vehicles for minimum fuel consumption over varying terrain," Trans. Res., Vol. 11, No. 4, pp. 279-286 (1977).

4. Saerens, B., "Optimal control based eco-driving theoretical approach and practical applications," Ph.D. Thesis, KU Leuven (2012).

5. Lattemann, F., K. Neiss, S. Terwen, and T. Connolly. The predictive cruise control - a system to reduce fuel consumption of heavy duty trucks. Technical Report 2004-01-2616, SAE, 2004.

6. Kamal, M., M. Mukai, J. Murata, and T. Kawabe, "Ecological vehicle control on roads with up-down slopes," IEEE Trans. Intell. Transp. Syst., Vol. 12, No. 3, pp. 783-794 (2011).

7. Kamal, M. A. S., M. Mukai, J. Murata, and T. Kawabe, "Model predictive control of vehicles on urban roads for improved fuel economy," IEEE Trans. Control Syst. Technol., Vol. 21, No. 3, pp. 831-841 (2013).

8. Asadi, B. and A. Vahidi, "Predictive cruise control: Utilizing upcoming traffic signal information for improving fuel economy and reducing trip time," IEEE Trans. Control Syst. Technol., Vol. 19, No. 3, pp. 707-714 (2011).

9. Lin, X, D. Gorges, and S. Liu, "Eco-driving assistance system for electric vehicles based on speed profile optimization," 2014 IEEE Conference on Control Applications (CCA), Antibes, France, pp. 629-634 (2014).

10. Li, X., Y. Chen, and J. Wang, "In-wheel motor electric ground vehicle energy management strategy for maximizing the travel distance," 20102 American Control Conference (ACC), Montreal, Canada, pp. 4993-4998 (2012).

11. Kuriyama, M., S. Yamamoto, and M. Miyatake, "Theoretical study on eco-driving technique for an electric vehicle with dynamic programming," 2010 International Conference on Electrical Machines and Systems (ICEMS), Incheon, South Korea, pp. 2026-2030 (2010).

12. Dib, W., L. Serrao, and A. Sciarretta, "Optimal control to minimize trip time and energy consumption in electric vehicles," 2011 IEEE Vehicle Power and Propulsion Conference (VPPC), Chicago, Illinois, USA, pp. 1-8 (2011).

13. Kirches, C., "Fast numerical methods for mixed-integer nonlinear model-predicitve control," Ph.D. Thesis, Ruprecht-Karls-Universität Heidelberg (2010).

14. Schwickart, T., H. Voos, J. R. Hadji-Minaglou, and M. Darouach, "A novel model-predictive cruise controller for electric vehicles and energy-efficient driving," AIM 2014. 11th IEEE International Conference on Advanced Intelligent Mechatronics, 2014, Besancon, France, pp. 1067-1072 (2014).

15. Schwickart, T., H. Voos, J.-R. Hadji-Minaglou, M. Darouach, and A. Rosich, "Design and simulation of a real-time implementable energy-efficient modelpredictive cruise controller for electric vehicles," J. Frankl. Inst.-Eng. Appl. Math., Vol. 352, No. 2, pp. 603-625 (2014).

16. Guzzella, L. and A. Sciarretta, Vehicle Propulsion Systems - Introduction to Modeling and Optimization, Springer, Berlin Heidelberg, Germany (2013).

17. Zomotor, A. and J. Reimpell, Fahrwerktechnik, Fahrverhalten, Vogel Verlag, Würzburg, Germany (1991).

18. Smart, Specifications (2013). (Accessed October 2013.) Available from: http://www.smart.de/. 
19. Hellstroem, E., "Look-ahead control of heavy vehicles," Ph.D. Thesis, Linköping University (2010).

20. Kohut, N., K. Hedrick, and F. Borrelli, "Integrating traffic data and model predictive control to improve fuel economy," 12th IFAC Symposium on Control in Transportation Systems, Redondo Beach, California, USA, pp. 155-160 (2009).

21. Saerens, B., H. A. Rakha, M. Diehl, and E. V. den Bulck, "A methodology for assessing eco-cruise control for passenger vehicles," Transp. Res. Part DTransp. Environ., Vol. 19, No. 0, pp. 20-27 (2013).

22. Stefanov, S., Separable Programming - Theory and Methods, Kluwer Academic Publishers, Dordrecht/ Boston/ London (2001).

23. Boyd, S. and L. Vandenberghe, Convex Optimization, Cambridge University Press, New York (2004).

24. Magnani, A. and S. Boyd, "Convex piecewise-linear fitting," Optim. Eng., Vol. 10, No. 1, pp. 1-17 (2009).

25. Mayne, D. Q., J. B. Rawlings, C. V. Rao, and P. O. Scokaert, "Constrained model predictive control: Stability and optimality," Automatica, Vol. 36, No. 6, pp. 789-814 (2000).

26. Maciejowski, J. M., Predictive Control with Constraints, Pearson Education, Prentice Hall, New York City, USA (2001).

27. Mattingley, J. and S. Boyd, "Cvxgen: a code generator for embedded convex optimization," Optim.Eng., Vol. 13, No. 1, pp. 1-27 (2012).

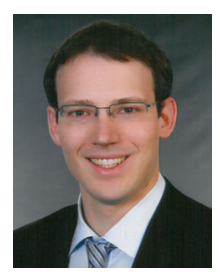

Tim Schwickart was born in 1985 in Hermeskeil, Germany. He received his Master degree in mechanical engineering at the Trier University of Applied Sciences in 2011 with a Master's thesis on the energy consumption estimation of vehicles on specific routes. Thereafter, he joined the University of Luxembourg as a Ph.D. student working on an energy-saving cruise control system for electric cars by means of model-predictive control. His research interests include driver-assistance-systems, model-predictive control, convex optimisation and optimal control.

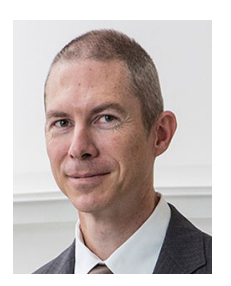

Holger Voos studied Electrical Engineering at the Saarland University and received the Doctoral Degree in Automatic Control from the Technical University of Kaiserslautern, Germany, in 2002. From 2000 to 2004, he was with Bodenseewerk Gerätetechnik $\mathrm{GmbH}$, Germany as Systems Engineer in aerospace and robotics. From 2004 to 2010, he was Professor at the University of Applied Sciences Ravensburg-Weingarten, Germany, and the head of the Mobile Robotics Lab there. Since 2010, he is Professor at the University of Luxembourg in the Faculty of Science, Technology and Communication, Research Unit of Engineering Sciences. He is the head of the Automatic Control Research Group and also the head of the Automation Lab in the Interdisciplinary Centre of Security, Reliability and Trust (SnT) at the University of Luxembourg. His research interests are in the area of distributed and networked control, model predictive control and safe and secure automation systems with applications in mobile robotics, energy systems and biomedicine.

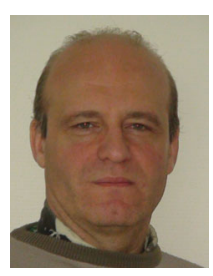

Jean-Régis Hadji-Minaglou was born in 1966 in Marseille, France. He received his MS degree in Electro-Mechanical Engineering at the École Nationale Supérieure d'Arts et Métiers (ENSAM -ParisTech) in Paris, France, in 1989 and his $\mathrm{PhD}$ degree in Electrical Engineering at the RWTH Aachen, Germany, in 1994. After a career in the manufacturing industry as Process Control Engineer he joined in 2007 the University of Luxembourg, Grand-Duchy of Luxembourg, where he is currently Full Professor in the Department of Electrical Energy, in charge of the chair for electrical drives. His research interests include renewable energies, decentralized electric energy production and integration of distributed electrical generation and consumption.

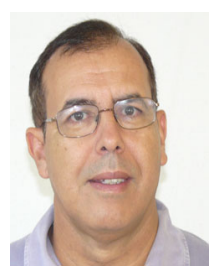

Mohamed Darouach graduated from "Ecole Mohammadia d'Ingénieurs", Rabat, Morocco,in 1978, and received the Doctor Engineer and Doctor of Sciences degrees from Nancy University, France, in 1983 and 1986, respectively. From 1978 to 1986 he was Associate Professor and Professor of automatic control at Ecole Hassania des Travaux Publics, Casablanca, Morocco. Since 1987 he is Professor at Université de Lorraine. He has been Vice Director of the Research Center in Automatic Control of Nancy (CRAN UMR 7039, Nancy-University, CNRS) from 2005 to 2013. He obtained a degree Honoris Causa from the Technical University of IASI and since 2010 he is a member of the Scientific council of Luxembourg University. Since July 2013, he is Vice Director of the University Institute of Technology of Longwy (Université de Lorraine). He held invited positions at University of Alberta, Edmonton. His research interests span theoretical control, observers design, and control of large-scale uncertain systems with applications. 